\title{
Mechanochemical Activation of Polymer-Embedded Photolumines- cent Benzoxazole Moieties
}

\author{
Marc Karman, ${ }^{\mathrm{a}}$ Ester Verde-Sesto, ${ }^{\mathrm{a}, \mathrm{b}}$ Christoph Weder ${ }^{\mathrm{a} *}$ \\ aAdolphe Merkle Institute, University of Fribourg, Chemin des Verdiers 4, 1700 Fribourg, Switzerland \\ ${ }^{\mathrm{b}}$ POLYMAT, University of the Basque Country UPV/EHU, Joxe Mari Korta Center, Avda. Tolosa 72, 20018 Donostia- \\ San Sebastián, Spain
}

\begin{abstract}
Despite an increasing number of studies that have investigated mechanochemical effects in polymers, the number of polymers whose fluorescence characteristics change upon exposure to mechanical stress is still limited. We here report the investigation of a mechanofluorophore based on an aliphatic ester of 2-(2'-hydroxyphenyl)benzoxazole. The free benzoxazole displays green photoluminescence, which is associated with an excited state intramolecular proton transfer (ESIPT) process, whereas aliphatic esters of this compound emit blue light. When poly(methyl acrylate) PMA containing an esterified benzoxazole mechanophore at the center of each chain molecule was exposed to ultrasound, a significant reduction of the molecular weight and pronounced changes of the photoluminescence emission and UV-Vis absorption spectra were observed. The optical changes and the fact that the time-traces for molecular weight decrease and formation of the ESIPT capable species mirror each other indicate that the mechanophore is preferentially cleaved upon sonication and that such cleavage restores the 2-(2'-hydroxyphenyl)benzoxazole motif. The concept of mechanical activation of ester-protected ESIPT dyes, and more broadly of other hydroxyl group carrying fluorophores that change their emission properties upon ester formation and cleavage, should be general and allow access to a range of other mechanofluorophores.
\end{abstract}

Mechanoresponsive polymers promise to be practically useful, as they enable functions such as mechanically activated catalysis, ${ }^{1}$ release of small molecules, ${ }^{2}$ selfstrengthening, ${ }^{3}$ and mechanically switchable electrical conductivity. ${ }^{4}$ Much of the research on mechanoresponsive polymers has been focused on the investigation of mechanochromic materials, in which deformation events cause a change of the absorption and/or emission color. ${ }^{5-7}$ Such materials are useful for fundamental research and also as damage or structural failure indicators. ${ }^{8,9}$ Several strategies have been devised to create mechanochromic materials, including the use of aggregachromic dyes that are blended into polymers and dispersed upon deformation, ${ }^{10-12}$ rupturing microcapsules, ${ }^{13-15}$ photonic structures, ${ }^{16,17}$ and the insertion of so-called mechanophores into macromolecules. ${ }^{18}$ The latter approach was first used by Moore and co-workers to create mechanochromic polymers, ${ }^{19}$ and has since been exploited in many polymer types and architectures, and in connection with a range of "mechanochromophores", i.e., mechanically labile motifs, which change their optical characteristics upon activation. ${ }^{20-22}$ Spiropyrans which transform into colored merocyanines is are the most widely used motifs to create mechanically induced absorption color changes, whereas dioxetane, tetraarylsuccinonitrile, cinnamoyl dimers, diaminodiphenyl-methane, anthracene maleimide adducts, and rhodamine, which function by homolytic cleavage, retro Diels-Alder reaction, and ring opening reaction, are known to change their fluorescence characteristics upon mechanochemical modification. ${ }^{20,23-27}$

Here we show a possibly broadly applicable approach to design photoluminescence changing mechanophores that relies on cleaving a weak ester bond coupled to a fluorophore, which changes its emission properties upon ester formation and cleavage. As a first example, we used a derivative of the photoluminescent 2-(2'-hydroxyphenyl)benzoxazole, whose luminescence in non-polar environments is associated with an excited state intramolecular proton transfer (ESIPT) ${ }^{28,29}$ Kocher et al. demonstrated that the fluorophore's green ESIPT emission can be switched off by esterification with aromatic acids, whereas derivatization with aliphatic esters shifts the emission to blue..$^{30}$ The ester's $\mathrm{C}-\mathrm{O}$ bonds are relatively weak, and this was previously exploited to use aromatic esters of 2(2'-hydroxyphenyl)benzoxazole as latent fluorophores, which can be activated by photochemical ester cleavage..$^{30}$ Speculating that a similar effect could also be achieved by way of mechanochemical activation, we devised a hitherto unknown benzoxazole featuring two hydroxyl groups (1), used the latter to incorporate the motif into a poly(methyl acrylate) (PMA) backbone, and tested the dissociation of the polymer-embedded mechanophores by exposing solutions of these molecules to ultrasound. This frequently used technique relies on the fact that the 
collapse of cavitation bubbles induces high shear forces, which stretch the polymer chains whose length is above a specific cutoff to a point where chemical bond cleavage occurs. $^{31}$ Gratifyingly, when the benzoxazole-containing polymer (Bz-PMA) was sonicated, the molecular weight decreased and the emission color changed, in a manner that is consistent with ester-bond cleavage and restoration of the ESIPT process.

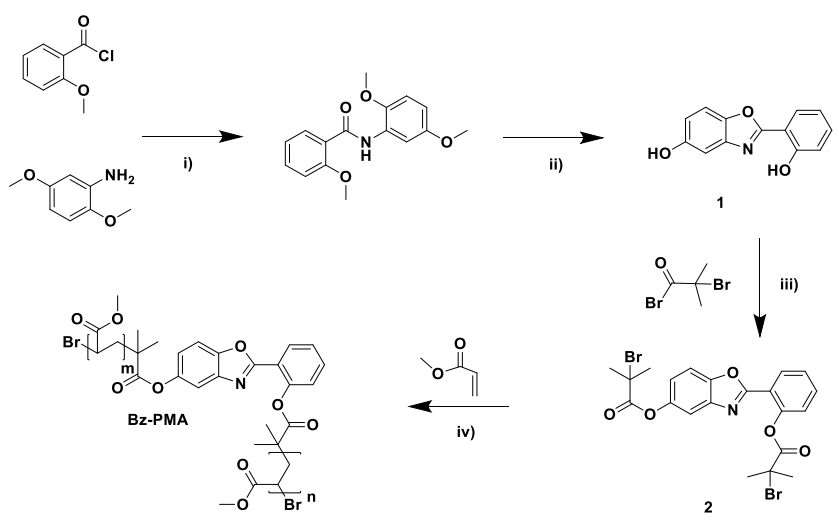

Scheme 1: Synthesis of N-(2,5-dimethoxyphenyl)-2-methoxybenzamide, 2-(2-hydroxyphenyl) benzoxazol-5-ol (1), the benzoxazole radical polymerization initiator (2), and the benzoxazole-containing PMA (Bz-PMA). i) $\mathrm{Et}_{3} \mathrm{~N}, \mathrm{THF}, \mathrm{o}^{\circ} \mathrm{C}$, 30 min., 92\%; ii) Pyridine, $\mathrm{HCl}, 200^{\circ} \mathrm{C}, 1 \mathrm{~h}, 64 \%$; iii) $\mathrm{Et}_{3} \mathrm{~N}$, THF (dry), r.t., overnight, 33\%; iv) $\mathrm{Cu}(\mathrm{o})$, Me6TREN, DMSO, r.t., overnight.

The choice of Bz-PMA (Scheme 1) as a first testbed was guided by the fact that in order to be preferentially activated, mechanophores must be placed near the center of the macromolecules, where they experience the highest force, ${ }^{21}$ and that PMA has previously been used in a range of mechanochemical studies. ${ }^{32-34}$ Thus, our synthetic route started with the Schotten-Baumann reaction between 2,5dimethoxyaniline and 2-methoxy-benzoyl chloride, which afforded (2,5-dimethoxy-phenyl)-2-methoxy-benzamide in good yield (92\%). The treatment of this product with pyridine hydrochloride at $200^{\circ} \mathrm{C}$ induced the ring closing reaction and deprotection of the methoxy group and afforded 2-(2-hydroxyphenyl) benzo[d]oxazol-5-ol (1) in appreciable yield (64\%). Finally, 2-(5-((2-bromo-2methylpropanoyl)oxy)benzo[d]oxazol-2-yl)phenyl-2-

bromo-2-methyl propanoate (2) was prepared via the double esterification of $\mathbf{1}$ with $\alpha$-bromoisobutyryl bromide (33\% yield). This specific modification was devised, because the $\alpha$-bromoisobutyryl groups can serve to initiate atom transfer radical polymerization from both ends of the $\mathrm{Bz}$ motif, which places one mechanophore at the center of each macromolecule. Further, prior work ${ }^{30}$ suggested that aliphatic (as opposed to aromatic) ester groups would keep the mechanophore emissive in the polymer-tethered form (but with a different emission color than the unmodified compound), so that the mechanoresponse would not be a turn-on effect (as would be expected for aromatic esters), but an emission color change. This is advantageous since a ratiometric signal is produced, which renders the system self-calibrating. ${ }^{13}$
The mechanophore-containing initiator 2 was employed for the supplemental activation reducing agent (SARA) atom transfer radical polymerization (ATRP) of methyl acrylate in DMSO, with $\mathrm{Cu}(\mathrm{o})$ and tris[2-(dimethylamino) ethyl]amine (Me ${ }_{6}$ TREN) as catalytic system. The Bz-PMA thus made had a number-average molecular weight $\left(M_{\mathrm{n}}\right)$ of $108.5 \mathrm{~kg} / \mathrm{mol}$ and a dispersity (Đ) of 1.19 . The polymer thus fulfills the requirements for ultrasound-induced chain-scission experiments, as the threshold $M_{\mathrm{n}}$ for sonochemical activation of neat PMA is lower $\left(87 \mathrm{~kg} / \mathrm{mol}^{33}\right)$ and this threshold is known to drop further upon incorporation of a mechanophore. ${ }^{35}$ To carry out control experiments, a Bz-free PMA (107.2 kg/mol, $Đ=1.13)$ was used as reference. ${ }^{34}$ In order to interpret the photophysical properties of Bz-PMA and the anticipated cleavage products, the model compounds 2-(2-hydroxy-phenyl) benzo[d]oxazol-5-yl acetate (3) and 2-(5acetoxybenzo[d]oxazol-2-yl)phenyl acetate (4) were prepared in addition to 1, 2, and Bz-PMA (Chart 1 ).
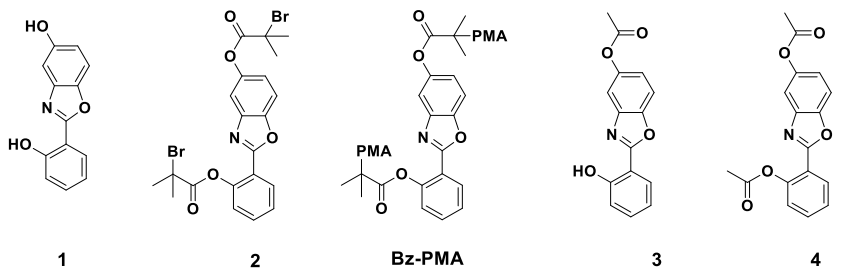

Chart 1: Structure of the different benzoxazole derivatives synthesized and used to elucidate the optical properties of the benzoxazole systems.

A comparison of the UV-Vis absorption spectra of compounds 1-4 and Bz-PMA (Figure 1a) shows clearly that the esterification of the hydroxyl group involved in the ESIPT process shifts the characteristic absorption band observed in 1 and 3 (broad with two peaks between 320 and $350 \mathrm{~nm}$ ) by about $30 \mathrm{~nm}$ to lower wavelengths (2, 4, Bz-PMA). This shift permits to preferentially (but not fully selectively) excite the species capable of ESIPT, and those that are not. A comparison of the absorption spectra of 1 and 3 shows that esterifying only the $\mathrm{OH}$ group that is not involved in the ESIPT has a much smaller influence, although the position of the bands (323 and 336 $\mathrm{nm}$ in the case of $\mathbf{3}, 332$ and $347 \mathrm{~nm}$ in the case of $\mathbf{1}$ ) is diagnostic for the species at hand (vide infra). Both molecules capable of ESIPT (1 and 3) display, as expected, green photoluminescence with an emission maximum of ca. $500 \mathrm{~nm}$ (Figure $\mathbf{1 b}$ ) and quantum yields (QY) of 0.071 and 0.037 , respectively. The green emission is absent in the other compounds. Intense blue emission with a maximum around $355 \mathrm{~nm}$ is observed in the case of $\mathbf{4}$ and $\mathbf{B z}$ PMA (QY= 0.14 and 0.21 ), whereas the initiator 2 displays a much weaker blue emission (maximum $=355 \mathrm{~nm}$ ), consistent with the presence of bromine atoms, which are known to stifle radiative processes by way of heavy atom effect. ${ }^{36}$ While one could lament about the fact that the quantum yields of the emitters used here are a bit low (lower quantum yields are typical for ESIPT processes), ${ }^{37}$ the various species display significant spectroscopic dif- 
ferences, which should be useful to monitor the mechanically induced response of the Bz-PMA.
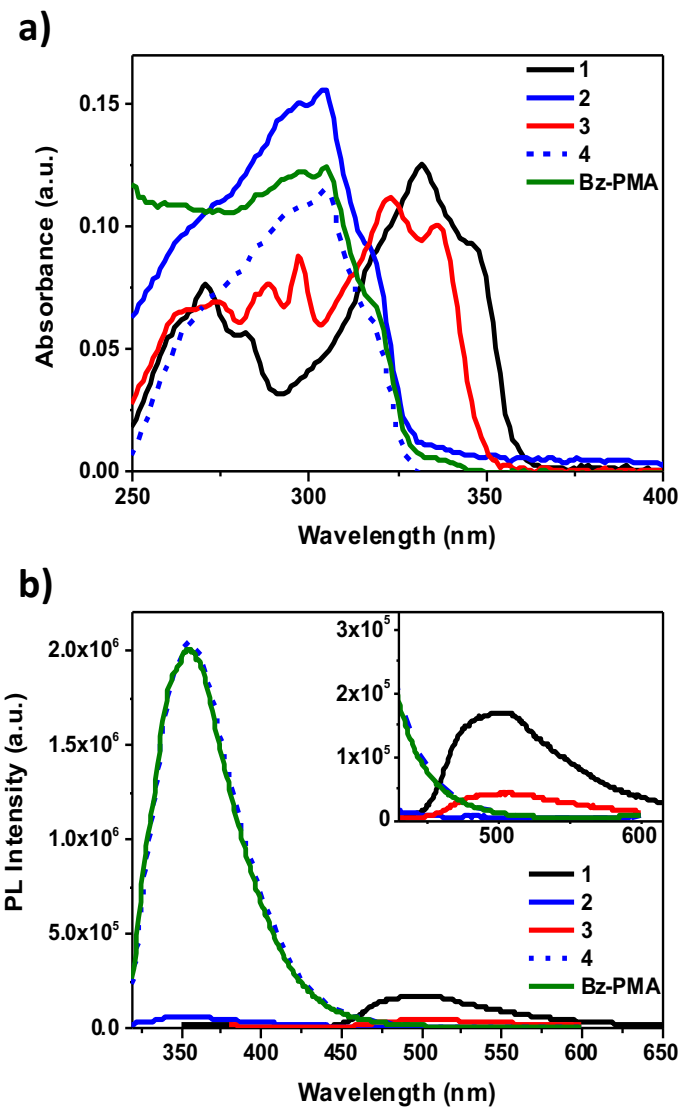

Figure 1: a) UV-Vis absorption and b) photoluminescence spectra of 1, 2, 3, 4, and Bz-PMA. All measurements were carried out in $10^{-5} \mathrm{~mol} \cdot \mathrm{L}^{-1}$ THF solutions (in case of Bz-PMA the concentration was based on the MA repeat unit) and the emission spectra were recorded with excitation at the respective absorption maximum. The inset in b) shows a magnification of the ESIPT region.

To investigate the mechanochemical activation of the $\mathrm{Bz}$ motif, dilute THF solutions of Bz-PMA were subjected to pulsed ultrasound $\left(10.4 \mathrm{~W} \cdot \mathrm{m}^{-2}\right)$ at $0^{\circ} \mathrm{C}$ over the course of 60 min. The effect of sonication was monitored throughout the course of the experiment by size-exclusion chromatography (SEC) and photoluminescence spectroscopy of aliquots that were taken at different time intervals. The SEC chromatograms of the sonicated Bz-PMA shows the expected development, with an initial decrease of the population of species corresponding to an $M_{\mathrm{n}}$ of 105 $\mathrm{kg} \cdot \mathrm{mol}^{-1}$ and a concomitant increase of a peak at a lower $M_{\mathrm{n}}$ of $59 \mathrm{~kg} \cdot \mathrm{mol}^{-1}$, indicating a gradual reduction of the polymer's molecular weight and splitting in the vicinity of the chains' centers (Figure 2a). The SEC traces recorded after longer sonication times further show a shoulder associated with an even lower $M_{\mathrm{n}}$ of $38 \mathrm{~kg} \cdot \mathrm{mol}^{-1}$, which is indicative of chains that are sufficiently long to undergo two scission events. A plot of the ratio of $M_{\mathrm{n}}$ and the initial number-average molecular weight $\left(M_{\mathrm{n}, \mathrm{o}}\right)$ as a function of ultrasonication time (Figure 2b, Figure S18) shows an exponential decay function to a level of $30 \%$ after 60 min.
A corresponding reference experiment with a mixture of the benzoxazole-free PMA and model compound 4 (Figure 2b, Figure S19, S2o) shows a qualitatively similar behavior, but the molecular weight decrease is substantially slower and the $M_{\mathrm{n}} / M_{\mathrm{n}, \mathrm{o}}$ curve levels off at only $42 \%$. Using the protocol reported by Lee et al. ${ }^{38}$ chain scission rate constants were determined for the Bz-PMA ( $k$ $\left.=2.8 \cdot 10^{-4} \mathrm{~min}^{-1} \mathrm{kDa}^{-1}\right)$ and the control polymer in presence of $4\left(k=2.0 \cdot 10^{-4} \mathrm{~min}^{-1} \mathrm{kDa}^{-1}\right.$, Figure S21 $)$. Thus, the data show that the mechanophore-containing polymer $\mathbf{B z}$ PMA is more easily cleaved upon exposure to ultrasound than the benzoxazole-free reference PMA.

a)

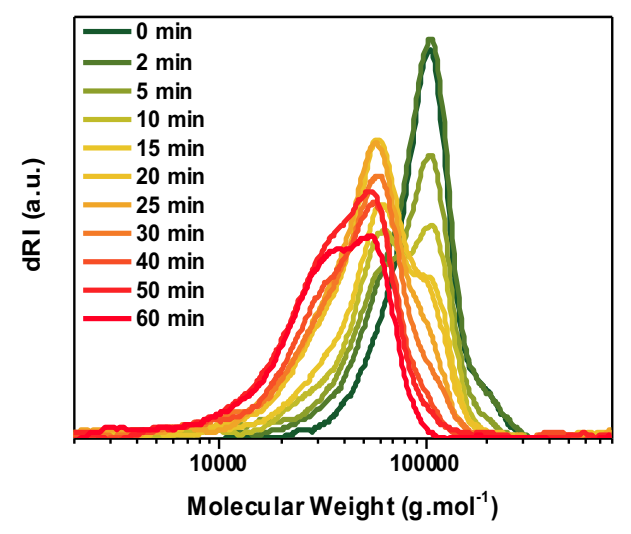

b)

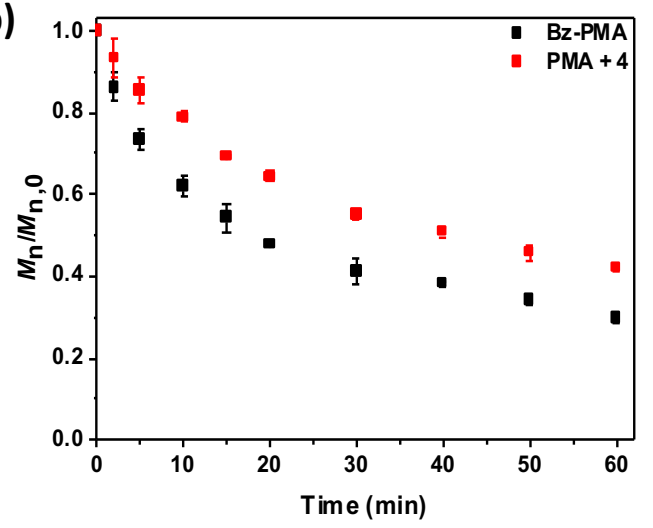

Figure 2: a) Representative size-exclusion chromatograms (based on refractive index signals) acquired upon ultrasonicating a THF solution of Bz-PMA $(\mathrm{c}=1 \mathrm{mg} / \mathrm{mL})$ for the times indicated. b) Evolution of the ratio of the number-average molecular weight $\left(M_{\mathrm{n}}\right)$ and the initial number-average molecular weight $\left(M_{\mathrm{n}, \mathrm{o}}\right)$ after ultrasonicating Bz-MPA and a reference system consisting of a concentration-matched mixture of PMA and 4 for the time indicated. Data shown are averages of $\mathrm{N}=3$ experiments in $1 \mathrm{mg} / \mathrm{mL}$ THF solutions.

In order to investigate if the mechanically induced cleavage of Bz-PMA occurs indeed at the mechanophore, samples of ultrasonicated solutions were not only subjected to SEC analysis (Figure 2), but also subjected to photoluminescence spectroscopy (Figure 3). In the case of Bz-PMA, the intensity of the blue photoluminescence associated with the intact mechanophore decreased as a function of sonication time, while concomitantly a broad emission band centered around $500 \mathrm{~nm}$, characteristic of ESIPT 
emission, appeared (Figure 3a). These changes, which can be visualized using a $450 \mathrm{~nm}$ long pass filter (Figure za inset), clearly support the conclusion that ultrasonica-
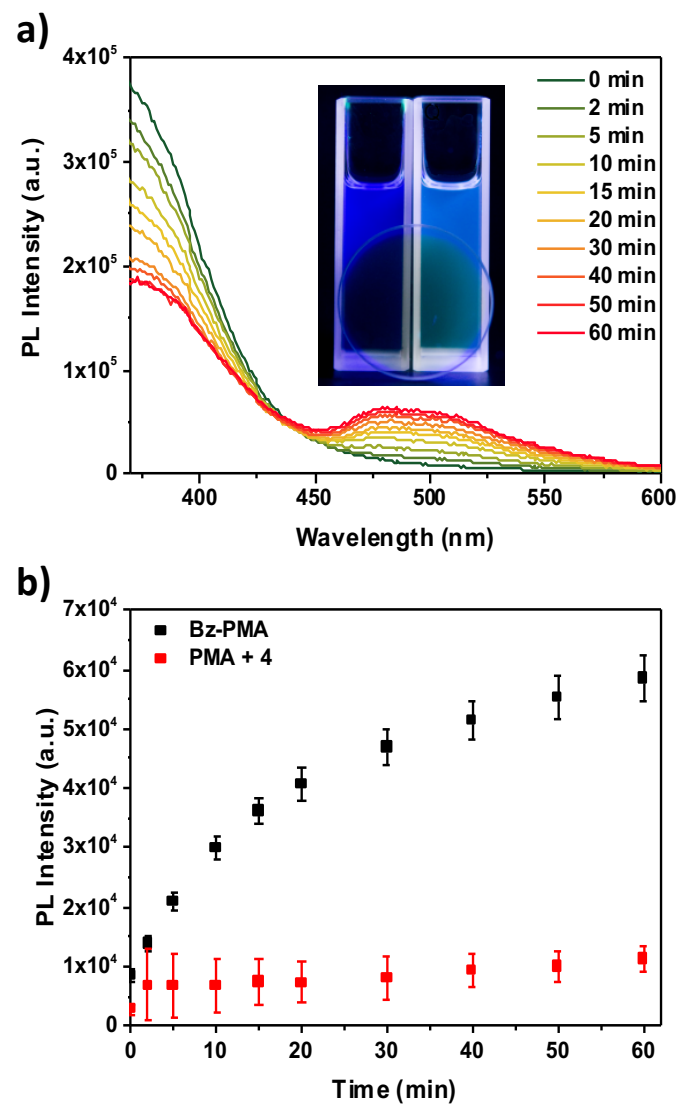

Figure 3: (a) Representative photoluminescence spectra $\left(\lambda_{\mathrm{ex}}\right.$ $=340 \mathrm{~nm}$ ) acquired upon ultrasonicating a dilute THF solution of Bz-PMA (c = $1 \mathrm{mg} / \mathrm{mL}$ ) for the times indicated. The inset shows a picture recorded under illumination with 365 $\mathrm{nm}$ UV light of the solution before (left) and after 6o min of sonication (right) using a $450 \mathrm{~nm}$ long pass filter. (b) Evolution of the emission intensity recorded at $500 \mathrm{~nm}\left(\lambda_{\mathrm{ex}}=340\right.$ $\mathrm{nm}$ ) acquired upon ultrasonicating a dilute THF solution of Bz-PMA (c = $1 \mathrm{mg} / \mathrm{mL}$, black squares) and a reference system consisting of a concentration-matched mixture of PMA and $\mathbf{4}$ (red squares) for the times indicated.

tion causes preferred cleavage of the ester function and restoration of the 2-hydroxy-phenyl moiety. Accordingly, the UV-Vis absorption spectrum of the Bz-PMA solution after exposure to ultrasound (Figure S22) shows two maxima at 335 and $320 \mathrm{~nm}$, which match the positions measured for model compound 3. No photoluminescence changes were observed upon ultrasonication of a solution containing Bz-free PMA and 4 (Figure $\mathbf{3} \mathbf{b}$, Figure S23, S24), which confirm that the restauration of the ESIPT emission upon ultrasonication of BZ-PMA require force transduction via the polymer backbone and that the mechanophore is not activated by reaction with products formed by unspecific chain scission. ${ }^{18}$ Gratifyingly, a plot of the photoluminescence intensity at $500 \mathrm{~nm}$ of a $\mathbf{B z}$ PMA solution as a function of sonication time (Figure $\mathbf{3} \mathbf{b}$, Figure S25) follows a function that mirrors that of the molecular weight decrease (Figure $\mathbf{2 b}$ ). In addition, the blue emission intensity was used to quantify the fraction of ester groups cleaved. A plot of the ratio of the photoluminescence intensity and the initial photoluminescence intensity against the sonication time shows an exponential decay function, which levels off at 50\% (Figure S26) suggesting that half of the benzoxazole moieties were activated. The facts that this level is somewhat lower than the $M_{\mathrm{n}} / M_{\mathrm{n}, \mathrm{o}}$ ratio (vide supra) and that the $M_{\mathrm{n}}$ decays at a faster rate than the photoluminescence intensities change indicate that the chain scission does not exclusively occur through ester cleavage, but also involves some unspecific events.

In summary, we have introduced a new mechanophore based on an aliphatic ester of 2-(2'-hydroxyphenyl)benzoxazole. When PMA containing this motif was exposed to ultrasound, pronounced changes of the photoluminescence emission and UV-Vis absorption spectra could be observed. Chain cleavage occurs preferentially at the mechanophore and restores the 2-(2'hydroxyphenyl)benzoxazole motif with high fidelity. In contrast to other mechanofluorochromic polymers that display a turn-on or turn-off behavior, the mechanophore investigated herein changes its photoluminescence color. The ratiometric signal thus produced is advantageous, as it is less dependent on experimental conditions (such as e.g. excitation intensity). However, the concept should also allow creating mechanophores with a turn-on effect, as aromatic esters of 2-(2'-hydroxyphenyl)benzoxazole were shown to be non-fluorescent. ${ }^{30}$ We further speculate that the concept of mechanical activation of esterprotected ESIPT dyes, and more broadly of other hydroxyl group carrying fluorophores that change their emission properties upon esterification and ester cleavage, should be general and that the motif presented here is only a first representative of a new class of mechanofluorophores.

\section{ASSOCIATED CONTENT}

The Supporting Information is available free of charge on the ACS Publications website at DOI: 10.1021/acsmacrolett.XXX. Experimental section including materials and methods, ${ }^{1} \mathrm{H}$ and ${ }^{13} \mathrm{C}$ NMR spectra, mass spectroscopy, elemental analysis results, melting point, SEC and photoluminescence data of sonication experiments.

\section{AUTHOR INFORMATION}

\section{Corresponding Author}

*E-mail: christoph.weder@unifr.ch

\section{ORCID}

Marc Karman: oooo-0003-0744-7625

Ester Verde-Sesto: oooo-0oo1-8748-5140

Christoph Weder: oooo-ooo1-7183-1790

Notes

The authors declare no competing financial interest.

\section{ACKNOWLEDGMENTS}


The authors thank Michela Di Giannantonio for providing the reference PMA. The research leading to these results has received funding from the European Research Council under the European Union's Seventh Framework Programme (FP7/2007-2013) / ERC grant agreement $\mathrm{n}^{\circ}$ [ERC-2011-AdG 291490-MERESPO]. This work was also partially supported by the Adolphe Merkle Foundation and the National Center of Competence in Research (NCCR) Bio-Inspired Materials, a research instrument of the Swiss National Science Foundation.

\section{REFERENCES}

1. Piermattei, A.; Karthikeyan, S.; Sijbesma, R. P., Activating catalysts with mechanical force, Nat. Chem. 2009, 1, 133-137.

2. Larsen, M. B.; Boydston, A. J., "Flex-activated" mechanophores: using polymer mechanochemistry to direct bond bending activation, J. Am. Chem. Soc. 2013, 135, 81898192.

3. Ramirez, A. L.; Kean, Z. S.; Orlicki, J. A.; Champhekar, M.; Elsakr, S. M.; Krause, W. E.; Craig, S. L., Mechanochemical strengthening of a synthetic polymer in response to typically destructive shear forces, Nat. Chem. 2o13, 5, 757-761.

4. Chen, Z.; Mercer, J. A. M.; Zhu, X.; Romaniuk, J. A. H.; Pfattner, R.; Cegelski, L.; Martinez, T. J.; Burns, N. Z.; Xia, Y., Mechanochemical unzipping of insulating polyladderene to semiconducting polyacetylene, Science 2017, 357, 475-479.

5. Calvino, C.; Neumann, L.; Weder, C.; Schrettl, S., Approaches to Polymeric Mechanochromic Materials, J. Polym. Sci. Pol. Chem. 2017, 55, 640-652.

6. Weder, C., Mechanochromic Polymers. In Encyclopedia of Polymeric Nanomaterials, Kobayashi, S. M., K. , Ed. Springer: 2015.

7. Sagara, Y.; Yamane, S.; Mitani, M.; Weder, C.; Kato, T., Mechanoresponsive Luminescent Molecular Assemblies: An Emerging Class of Materials, Adv. Mater. 2016, 28, 1073-1095.

8. Li, M.; Zhang, Q.; Zhou, Y. N.; Zhu, S. P., Let spiropyran help polymers feel force!, Prog. Polym. Sci. 2018, 79, 26-39.

9. Boulatov, R., The Challenges and Opportunities of Contemporary Polymer Mechanochemistry, ChemPhysChem 2017, 18, 1419-1421.

10. Kunzelman, J.; Crenshaw, B. R.; Kinami, M.; Weder, C., Selfassembly and dispersion of chromogenic molecules: A versatile and general approach for self-assessing polymers, Macromol. Rapid. Comm. 20o6, 27, 1981-1987.

11. Lowe, C.; Weder, C., Oligo(p-phenylene vinylene) excimers as molecular probes: Deformation-induced color changes in photoluminescent polymer blends, Adv. Mater. 2002, 14, 1625-1629.

12. Crenshaw, B. R.; Weder, C., Deformation-induced color changes in melt-processed photoluminescent polymer blends, Chem. Mater. 2003, 15, 4717-4724.

13. Calvino, C.; Guha, A.; Weder, C.; Schrettl, S., Self-Calibrating Mechanochromic Fluorescent Polymers Based on Encapsulated Excimer-Forming Dyes, Adv. Mater. 2018, 30, 1704603 .

14. Robb, M. J.; Li, W.; Gergely, R. C. R.; Matthews, C. C.; White, S. R.; Sottos, N. R.; Moore, J. S., A Robust Damage-Reporting Strategy for Polymeric Materials Enabled by AggregationInduced Emission, ACS Cent. Sci. 2016, 2, 598-603.

15. Lavrenova, A.; Farkas, J.; Weder, C.; Simon, Y. C., Visualization of Polymer Deformation Using Microcapsules Filled with Charge-Transfer Complex Precursors, ACS Appl. Mater. Interfaces 2015, 7, 21828-21834.

16. Akamatsu, N.; Hisano, K.; Tatsumi, R.; Aizawa, M.; Barrett, C. J.; Shishido, A., Thermo-, photo-, and mechano-responsive liquid crystal networks enable tunable photonic crystals, Soft Matter 2017, 13, 7486-7491.

17. Schafer, C. G.; Gallei, M.; Zahn, J. T.; Engelhardt, J.; Hellmann, G. P.; Rehahn, M., Reversible Light-, Thermo-, and Mechano-Responsive Elastomeric Polymer Opal Films, Chem. Mater. 2013, 25, 2309-2318.

18. Li, J.; Nagamani, C.; Moore, J. S., Polymer mechanochemistry: from destructive to productive, Acc. Chem. Res. 2015, 48, 2181-219o.

19. Davis, D. A.; Hamilton, A.; Yang, J.; Cremar, L. D.; Van Gough, D.; Potisek, S. L.; Ong, M. T.; Braun, P. V.; Martinez, T. J.; White, S. R.; Moore, J. S.; Sottos, N. R., Force-induced activation of covalent bonds in mechanoresponsive polymeric materials, Nature 2009, 459, 68-72.

20. Chen, Y.; Spiering, A. J.; Karthikeyan, S.; Peters, G. W.; Meijer, E. W.; Sijbesma, R. P., Mechanically induced chemiluminescence from polymers incorporating a 1,2dioxetane unit in the main chain, Nat. Chem. 2012, 4, 559562.

21. Kean, Z. S.; Gossweiler, G. R.; Kouznetsova, T. B.; Hewage, G. B.; Craig, S. L., A coumarin dimer probe of mechanochemical scission efficiency in the sonochemical activation of chaincentered mechanophore polymers, Chem. Commun. 2015, 51, 9157-916o.

22. Imato, K.; Nishihara, M.; Irie, A.; Takahara, A.; Otsuka, H., Diarylbibenzofuranone-Based Dynamic Covalent Polymer Gels Prepared via Radical Polymerization and Subsequent Polymer Reaction, Gels 2015, 1, 58-68.

23. Sumi, T.; Goseki, R.; Otsuka, H., Tetraarylsuccinonitriles as mechanochromophores to generate highly stable luminescent carbon-centered radicals, Chem. Commun. 2017, 53, $11885-11888$.

24. Nofen, E. M.; Zimmer, N.; Dasgupta, A.; Gunckel, R.; Koo, B.; Chattopadhyay, A.; Dai, L. L., Stress-sensing thermoset polymer networks via grafted cinnamoyl/cyclobutane mechanophore units in epoxy, Polym. Chem.-Uk 2016, 7, 7249-7259.

25. Li, Z.; Toivola, R.; Ding, F.; Yang, J.; Lai, P. N.; Howie, T.; Georgeson, G.; Jang, S. H.; Li, X.; Flinn, B. D.; Jen, A. K., Highly Sensitive Built-In Strain Sensors for Polymer Composites: Fluorescence Turn-On Response through Mechanochemical Activation, Adv. Mater. 2016, 28, 65926597.

26. Gostl, R.; Sijbesma, R. P., pi-extended anthracenes as sensitive probes for mechanical stress, Chem. Sci. 2016, 7 , 370-375.

27. Wang, Z.; Ma, Z.; Wang, Y.; Xu, Z.; Luo, Y.; Wei, Y.; Jia, X., A Novel Mechanochromic and Photochromic Polymer Film: When Rhodamine Joins Polyurethane, Adv. Mater. 2015, 27, 6469-6474.

28. Das, K.; Sarkar, N.; Ghosh, A. K.; Majumdar, D.; Nath, D. N.; Bhattacharyya, K., Excited-State Intramolecular ProtonTransfer in 2-(2'-Hydroxyphenyl)Benzimidazole and 2-(2'Hydroxyphenyl)-Benzoxazole - Effect of Rotamerism and Hydrogen-Bonding, J. Phys. Chem.-Us 1994, 98, 9126-9132.

29. Woolfe, G. J.; Melzig, M.; Schneider, S.; Dörr, F., The role of tautomeric and rotameric species in the photophysics of 2(2'-hydroxyphenyl)benzoxazole, Chem. Phys. 1983, 77, 213221.

3o. Kocher, C.; Smith, P.; Weder, C., Aromatic 2-(2'hydroxyphenyl)benzoxazole esters: a novel class of caged photoluminescent dyes, J. Mater. Chem. 2002, 12, 2620-2626.

31. May, P. A.; Moore, J. S., Polymer mechanochemistry: techniques to generate molecular force via elongational flows, Chem. Soc. Rev. 2013, 42, 7497-7506.

32. May, P. A.; Munaretto, N. F.; Hamoy, M. B.; Robb, M. J.; Moore, J. S., Is Molecular Weight or Degree of 
Polymerization a Better Descriptor of Ultrasound-Induced Mechanochemical Transduction?, ACS Macro Lett. 2016, 5, 177-18o.

33. Potisek, S. L.; Davis, D. A.; Sottos, N. R.; White, S. R.; Moore, J. S., Mechanophore-linked addition polymers, J. Am. Chem. Soc. 2007, 129, 13808-13809.

34. Di Giannantonio, M.; Ayer, M. A.; Verde-Sesto, E.; Lattuada, M.; Weder, C.; Fromm, K. M., Triggered metal ion release and oxidation: Ferrocene as new mechanophore in polymers, Angew. Chem., Int. Ed. 2018, doi:10.1002/anie.201803524.

35. Kryger, M. J.; Munaretto, A. M.; Moore, J. S., Structuremechanochemical activity relationships for cyclobutane mechanophores, J. Am. Chem. Soc. 2011, 133, 18992-18998.
36. Rae, M.; Fedorov, A.; Berberan-Santos, M. N., Fluorescence quenching with exponential distance dependence: Application to the external heavy-atom effect, J. Chem. Phys. 2003, 119, 2223-2231.

37. Kwon, J. E.; Park, S. Y., Advanced organic optoelectronic materials: harnessing excited-state intramolecular proton transfer (ESIPT) process, Adv. Mater. 2o11, 23, 3615-3642.

38. Lee, B.; Niu, Z.; Wang, J.; Slebodnick, C.; Craig, S. L., Relative Mechanical Strengths of Weak Bonds in Sonochemical Polymer Mechanochemistry, J. Am. Chem. Soc. 2015, 137, 10826-10832. 
TOC Entry

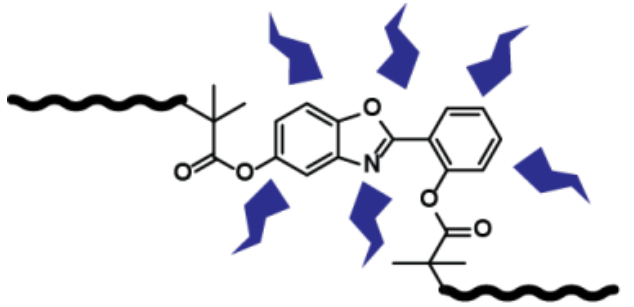

ESIPT OFF
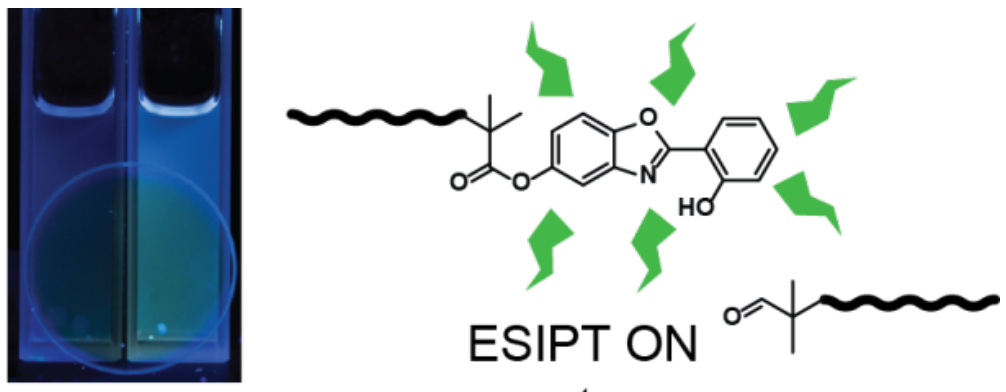

Ultrasonication Induced Mechanical Stress 